\title{
Integrated Adolescent Medicine Care Path for Pediatric Patients Diagnosed with Comorbid Obesity-Depression
}

Keywords: Obesity; Comorbid depression; Psychologist; Teachback method; Nutritionist

Abbreviations: PCP: Primary Care Physician; DBH-BHC: Doctor of Behavioral Health - Behavioral Health Consultant; PHQ-A: Patient Health Questionnaire for Adolescents

\section{Opinion}

A pediatric patient is diagnosed with Comorbid Depression and Obesity by Primary Care Physician (PCP) and handed off to the Doctor of Behavioral Health - Behavioral Health Consultant (DBHBHC). Initial behavioral health consult will include Motivational Interviewing and Teach-Back Method [1]. The doctor of behavioral health, pediatrician, nutritionist, and physical therapist then work together to provide effective treatment for the depressed and clinically obese adolescent patient and family [2]. Possible outside referral to include child psychologist, depending on treatment adherence and patient progress [3]. The following 3 SMART Goals and Objectives will be followed to move the patient's BMI, out of the obesity range, to below the 95th percentile and lower the patient's depression at least one level as measured by Patient Health Questionnaire for Adolescents (PHQ-A).

\section{Patient-Centered SMART Physical Activity (PA) goal; completed by adolescent}

S: The obese pediatric patient will complete 60 minutes of Cardio PA 5 days a week [4].

M: After 2 weeks the pediatric patient will lower their presence and severity of obesity as assessed by the BMI child measurement from obese category to overweight category.

A: After the referral from Pediatrician and during the BHC Intervention Interview, the adolescent patient and parent will fill out the consent form, personal information patient form, and attainable SMART goals PA treatment adherence contract.

R: Patient rates this as a 7 on scale of 1-10 of likelihood they will complete.

T: Patient will complete PA Program for 2 weeks; will review in 2 weeks with BHC.

Patient-Centered SMART PA weight training goal; completed with physical therapist

S: The obese pediatric patient will complete weight training PA with PT 2 days a week.

Volume 9 Issue 6 - 2017
Robelyn Garcia*
Arizona State University, USA
*Corresponding author: Robelyn Garcia, Arizona State
University, Scottsdale, AZ 85260, USA, Tel: 6028459802;
Email: Dr.RobelynGarcia@asu.edu
Received: November 21, 2016 | Published: December 07,
2017

M: After 8 weeks the pediatric patient will lower their presence and severity of depression as assessed by the PHQ-A by at least one category. BHC recommendations will follow.

A: During the BHC Interview, the patient and parent will sign the PT adherence contract.

R: During the 8-week PA Program the BHC and PT will monitor weekly patient progress.

T: Patient will complete PA Program for 8 weeks. Will review every 2 weeks with BHC.

Family-Centered SMART Diet Goal; to be signed by parent, child and nutritionist [5]

S: For breakfast at home, parent will replace child's pastry with one banana.

M: First, parent will remove the pastry from the house.

A: Parent will buy bananas at the store so they will be ready for child in the morning.

R: Patient and Parent rate this as 8 likelihood on scale of 1-10 they will complete.

T: Every day for a month and then evaluated by the BHC and nutritionist for new goal [6].

Acknowledgment

None.

\section{Conflict of Interest}

None. 


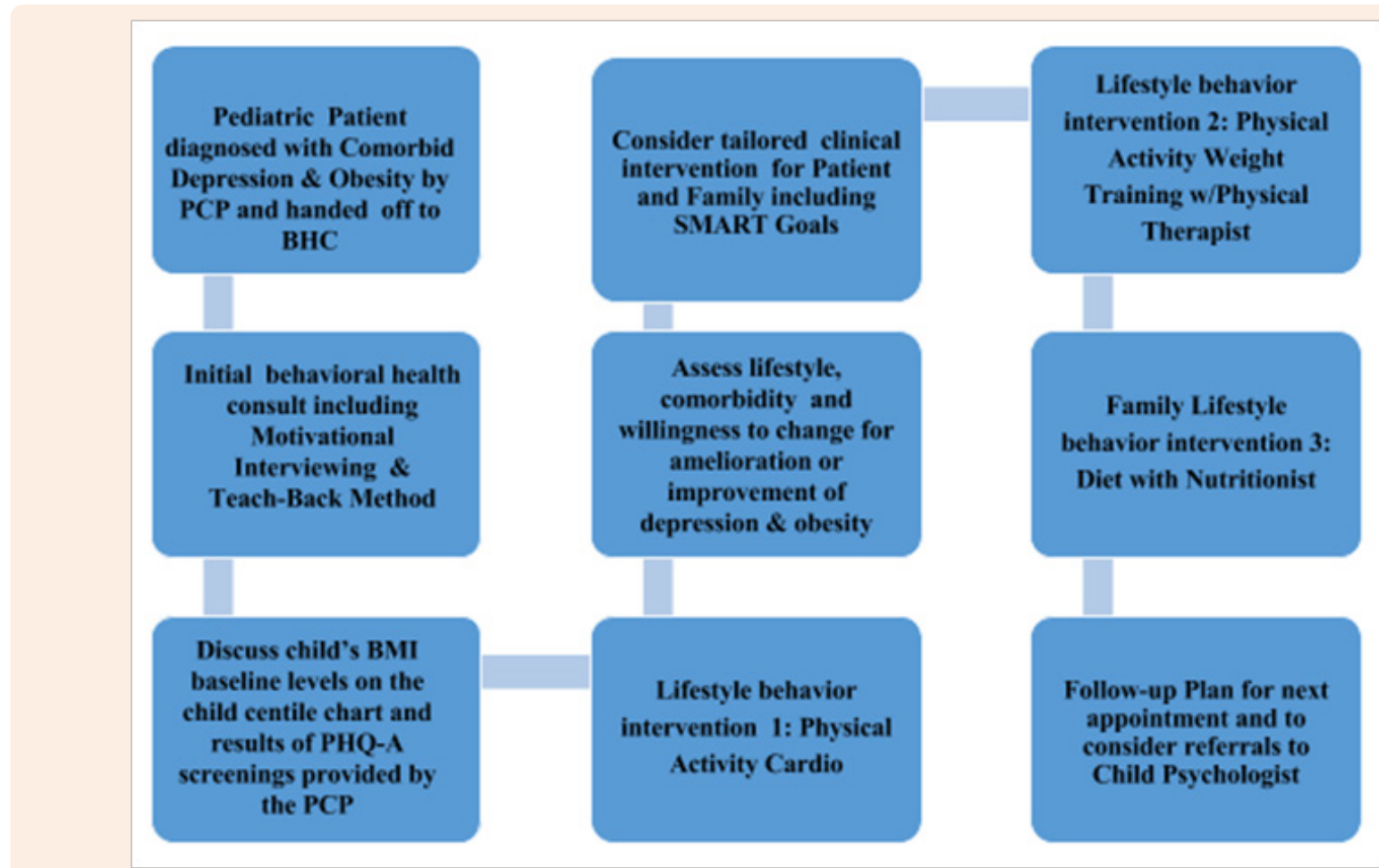

Figure 1: Adolescent Care Path.

\section{References}

1. UnityPoint Health (2017) Always Use Teach Back! Institute for Healthcare.

2. Garcia R, Benavidez D (2016) Transtheoretical Model Key Constructs Applied to The Intervention \& Treatment of Weight Cycling \& Yoyo Dieting Cognitive Affective Bases of Health for Weight Management. Int J Complement Alt Me 3(3): 00071.

3. Barlow SE, AAP Expert Committee (2007) Expert Committee Recommendations Regarding the Prevention, Assessment, and Treatment of Child and Adolescent Overweight and Obesity: Summary Report. Pediatrics 120(4): S164-S192.
4. Centers for Disease Control and Prevention (CDC) (2017) Increasing Physical Education and Physical Activity: A Framework for Schools.

5. GBS Corporate Training (2013) SMART objectives. [Video file].

6. Styne DM, Arslanian SA, Connor EL, Farooqi IS, Murad MH, et al. (2017) Pediatric Obesity-Assessment, Treatment, and Prevention: An Endocrine Society Clinical Practice Guideline. J Clin Endocrinol Metab 102(3): 709-757. 